\title{
Gamification, Persuasive Techniques, and Nudging: What is the Impact on the User Experience?
}

\author{
Olga De Troyer \\ WISE Research Lab, \\ Vrije Universiteit Brussel \\ Pleinlaan 2, 1050 Brussel - Belgium \\ Olga. DeTroyer@vub.be
}

\begin{abstract}
An important challenge faced by many software designers is how they can make users "hooked" to their software, i.e., what can they do to ensure that users continue to use their application over time, especially when there is no obligation to do so. Many apps suffer from the novelty effect and are abandoned quickly. Therefore, there is an increased interest in existing methods, techniques, and theories from different fields (such as psychology, social science, games) that can be used to keep users motivated to use the software. Nowadays, most popular are gamification, persuasive techniques, and nudging, all having their own characteristics and merits. We investigate these techniques from a user experience perspective. When can they be used successfully, when can they backfire? How can they be incorporated into software and are there ethical considerations? This paper is an extended abstract of an invited talk given at RoCHI 2021.
\end{abstract}

\section{Author Keywords}

Gamification; Persuasion; Nudging; User Experience; Personalization; Personality types, Gamification User Types.

\section{ACM Classification Keywords}

H.5.m. Information interfaces and presentation (e.g., HCI): Miscellaneous.

\section{General Terms}

Human Factors; Design.

DOI: 10.37789/rochi.2021.1.1.1

\section{EXTEDED ABSTRACT}

Some software products are a huge success and people keep using them, while others start as a hype but eventually die out, and still others are simply quickly abandoned. Therefore, an important challenge faced by many software designers is how they can make users "hooked" to their software, i.e., what can they do to ensure that users continue to use the application (i.e., achieve long term user retention, also called stickiness [1] being the extent to which customers remain engaged with an application, product or website), especially when there is no obligation to do so. For an application with a business purpose, the reason for aiming to achieve stickiness is mainly economic. For instance, the more users for a web shop and the more they keep coming back, the higher the profit. However, some applications are not created to make a direct profit. The profit can be indirect by engage people in a certain activity or in adopting a certain behavior. For instance, an abundance of apps has emerged for improving health through stimulating healthy behavior, to stimulate civic engagement, to help people reducing their ecological footprint, or to induce some attitude change. These apps often suffer from the novelty effect and are abandoned quickly.

Providing the right functionality is not sufficient to realize stickiness. Good marketing may help but can in general also not prevent the novelty effect. Therefore, there is an increased interest in existing methods, techniques, and theories from different fields (such as psychology, social science, marketing) that could be used within the software to keep users engaged and motivated to use the software. Nowadays, most popular are gamification, persuasive techniques, and nudging, all having their own characteristics and merits.

To understand how these techniques can work for realizing stickiness, we first need to understand the concepts of motivation and engagement. Both, motivation, and engagement can be defined in multiple ways $[2,3]$. In this work, we define motivation as the reason for the user's initial and sustained engagement in the activity [3, 4]. Different definitions have been given for engagement. In a well-cited article on user engagement [5], engagement is defined as "a category of user experience characterized by attributes of challenge, positive affect, endurability, aesthetic and sensory appeal, attention, feedback, variety/novelty, inter-activity, and perceived user control". In [6], user engagement is defined as "the emotional, cognitive and behavioral connection that exists, at any point in time and possibly over time, between a user and a resource". In the context of Digital Behavior Change Interventions (DBCI) [2], engagement with DBCI is defined as "(1) the extent (e.g., amount, frequency, duration, depth) of usage and (2) a subjective experience characterized by attention, interest and affect".

An important model in the context of behavior is the Behavioral Model of Fogg [7]. It gives three factors that determine whether a person will perform a certain behavior or not: motivation, ability, and trigger. According to Fogg, motivation can be distilled to three pairs of core motivators: pleasure and pain; hope and fear; and social acceptance and 
social rejection. These are aspects that could be taken into consideration in the development of tools to achieve stickiness, for instance, by providing pleasure, or use social acceptance as motivator. Fogg's ability relates to available resources and are defined in a broad sense, i.e., available time and/or money; required physical effort and/or cognitive effort; social deviance caused by the behavior; and the familiarity with the behavior. The trigger in Fogg's model is the element that sparks, facilitates, or signals the target behavior. The type of trigger used, as well as the content of the trigger, seems to be important. If an app keeps sending notifications, this might annoy the user and cause dropout.

Next, the Hook Model of Eyal [8] is a practical approach to create new behavior. According to Eyal, the reason why new behaviors do not last is because most behaviors are purely done out of habit. Turning a new behavior into a habit is difficult because old habits die hard, while new habits quickly dissipate. Therefore, the Hook Model proposes a cycle through which the user must repeatedly go to gradually create this new habit. A single cycle starts with a trigger that should be followed by an action from the user. This should be something simple and easy (based on Fogg's ability factor), e.g., clicking on a link. In the next phase, the user should be rewarded for the action to increase the likelihood of repeating the action in the next cycle. The last phase of the cycle is the investment, which is typical for the Hook Model. The principle is that the more a user invests in a system, the less likely it is that (s)he will stop using the system.

The triggers in both the Behavior Model of Fogg and the Hook Model of Eyal aim to persuade the user to perform a certain behavior or action. This brings us to persuasive technology. Persuasion is used to influence decisions or actions taken by human beings by applying certain psychological principles [9]. These principles use the fact that most of the time people do not take decisions or actions based on rational arguments but use shortcuts. Well known examples of principles of persuasion are those formulated by Cialdini [10]. When persuasion is used in ICT is it called persuasive technology [11].

Nudging is related to persuasion but generally adopts a pushier approach. A nudging system gently but firmly pushes the user to a certain behavior. Nudging was introduced in [12] and defined as "any aspects of the choice architecture that alters people's behavior in a predictable way without forbidding any options or significantly changing their economic incentives". It is often used in policy making [13] and health care.

Whereas persuasive technology and nudging aim to influence the behaviors or decisions of users, gamification aims to improve the user's experience through the use of game elements in a nongame context [14]. Gamification tries to tap into the intrinsic motivation of people to have fun. This principle is also use by serious games, which are characterized as (digital) games used for purposes other than mere entertainment [15]. Many game elements exist but only some are used for gamification, mainly badges, points and leaderboards [16].

Although gamification, persuasive technology and nudging seems promising for stimulating engagement, the question remains how effective these techniques are. To investigate this, we did a meta literature review on these three techniques [16]. For gamification, most papers reported positive findings. However, we also notice shortcomings in the user studies performed, like small sample sizes, lack of validated measurement instruments, lack of control groups, short time evaluations, and the use of descriptive statistics only. Furthermore, authors seem to be reluctant to report negative findings, and the impact of individual game elements remains unclear. For persuasive technology, reported findings were positive or partially positive. The work in this domain suffers from the same shortcomings as the work on gamification, and the same observations about publication bias and impact of individual techniques can be made. The finding for nudging were limited, as only two reviews were found for nudging: one being positive and one less positive.

The mixed results could be explained by the fact that people are different: they have different preferences, different abilities, different levels of motivations, and different personality treats. What is engaging and effective for one person may not be engaging and effective for another person. Therefore, a solution could be to personalize the techniques used in an application. By considering the needs, abilities, preferences, and traits of the individual user, one could possibly affect the effectiveness of the approaches. To be able to offer a personalized approach, one must first know the user, i.e., understand the user's needs, abilities, and preferences, and then adapt or construct the product and interventions accordingly. Next to the well-known user aspects considered as inputs for the personalization, such as needs and preferences, also personality traits could be interesting to consider. Results on the relationship between personality traits and the effectiveness of gamification, serious games, or persuasive technology has already been reported [17-26]. To realize such a personalized application, often an adaptive engine is used driven by some form of "adaptation rules" and using information about the user usually stored in a user profile, possibly complimented with a personality profile, and/or a persuasion profile [19]. Also, the runtime behavior of the user can be monitored and tracked to further enhance the personalization and to improve and adjust the personality and persuasion profile. Instead of rules also some form of artificial intelligence can be applied. Related to such far-reaching personalization is the ethics of collecting and maintaining so much information about a user. What about the privacy of the user, and how much can the user trust that this information will not be misused? The GDPR law, a legal framework for the collection and processing of personal information from individuals living in the European Union, provides a legal context, but is this sufficient to protect users from misuse of their data? 


\section{REFERENCES}

1. J. C. C. Lin, "Online stickiness: its antecedents and effect on purchasing intention," Behav. Inf. Technol., vol. 26, no. 6, pp. 507-516, 2007.

2. O. Perski, A. Blandford, R. West, and S. Michie, "Conceptualising engagement with digital behaviour change interventions: a systematic review using principles from critical interpretive synthesis.," Transl. Behav. Med., vol. 7, no. 2, pp. 254-267, 2017.

3. T. H. Laine and R. Lindberg, "Designing Engaging Games for Education: A Systematic Literature Review on Game Motivators and Design Principles," IEEE Trans. Learn. Technol., vol. 13, no. 4, pp. 804-821., 2020.

4. R. M. Ryan and E. L. Deci, "Intrinsic and extrinsic motivations: Classic definitions and new directions.," Contemp. Educ. Psychol., vol. 25, no. 1, pp. 54-67, 2000.

5. H. L. O'Brien and E. G. Toms, "What is user engagement? A conceptual framework for defining user engagement with technology.," J. Am. Soc. Inf. Sci. Technol., vol. 59, no. 6, pp. 938-955, 2008.

6. S. Attfield, G. Kazai, M. Lalmas, and B. Piwowarski, "Towards a science of user engagement (position paper)," in WSDM workshop on user modelling for Web applications, 2011, pp. 9-12.

7. B. Fogg, "A Behavior Model for Persuasive Design," in Proceedings of the 4th International Conference on Persuasive Technology - Persuasive '09, 2009, p. 40.

8. N. Eyal, Hooked: How to Build Habit-Forming Products. London: Penguin Books Ltd., 2014.

9. H. W. Simons, J. G. Jones, and H. W. Simons, Persuasion in society. Routledge, NY, USA, 2017.

10. N. J. Goldstein, M. J. Steve, and R. B. Cialdini, Yes! 50 Scientifically proven ways to be persuasive. New York: Simon \& Schuster Inc., 2008.

11.B. J. Fogg, "Persuasive technology: using computers to change what we think and do," Ubiquity, vol. 2002, no. December, p. 5, 2002.

12. R. Thaler and C. R. Sunstein, Nudge: Improving decisions about health, wealth, and happiness. Yale University Press, 2008.

13.P. G. Hansen and A. M. Jespersen, "Nudge and the manipulation of choice: A framework for the responsible use of the nudge approach to behaviour change in public policy," Eur. J. Risk Regul., vol. 4, no. 1, pp. 3-28, 2013.

14. S. Deterding, D. Dixon, R. Khaled, and L. Nacke, "From game design elements to gamefulness: Defining gamification," in Proceedings of the 15th International Academic MindTrek Conference on Envisioning Future Media Environments - MindTrek '11, 2011.

15. M. Zyda, "From visual simulation to virtual reality to games," Computer (Long. Beach. Calif)., vol. 38, no. 9, pp. 25-32, 2005.

16. R. Lindberg, T. H. Laine, and O. De Troyer, "Gamification, Persuasive Technology and Nudging: A Systematic Meta Review,” 2021 (under review).

17. M. Kaptein, J. Lacroix, and P. Saini, "Individual differences in persuadability in the health promotion domain," in Persuasive technology, Springer, 2010, pp. 94-105.

18. M. Kaptein, B. De Ruyter, P. Markopoulos, and E. Aarts, "Adaptive Persuasive Systems," ACM Trans. Interact. Intell. Syst., vol. 2, no. 2, pp. 1-25, 2012.

19. P. Markopoulos, M. Kaptein, B. De Ruyter, and E. Aarts, "Personalizing persuasive technologies: explicit and implicit personalization using persuasion profiles," Int. J. Hum. Comput. Stud., vol. 77, pp. 38-51, 2015.

20. M. Kaptein, M. Panos, B. de Ruyter, and E. Aart, "Can You Be Persuaded? Individual Differences in Susceptibility to Persuasion," in INTERACT 2009, 2009, pp. $115-118$.

21. M. Busch et al., "Personalization in Serious and Persuasive Games and Gamified Interactions," in Proceedings of the 2015 Annual Symposium on Computer-Human Interaction in Play, 2015, pp. 811816.

22. R. Orji, J. Vassileva, and R. L. Mandryk, "Modeling the efficacy of persuasive strategies for different gamer types in serious games for health," User Model. Useradapt. Interact., vol. 24, no. 5, pp. 453-498, 2014.

23. R. Orji, R. L. Mandryk, and J. Vassileva, "Gender, Age, and Responsiveness to Cialdini's Persuasion Strategies," Persuas. Technol., pp. 147-159, 2015.

24. S. Halko and J. A. Kientz, "Personality and persuasive technology: an exploratory study on health-promoting mobile applications," in Persuasive technology, Springer, 2010, pp. 150-161

25.P. Sajjadi, J. Vlieghe, and O. De Troyer, "Evidencebased mapping between the theory of multiple intelligences and game mechanics for the purpose of player-centered serious game design," in Games and Virtual Worlds for Serious Applications (VS-Games), 2016 8th International Conference on, 2016, pp. 1-8.

26. A. C. G. Santos et al., "The relationship between user types and gamification designs," User Model. Useradapt. Interact., 2021. 